\title{
Review: antibiotics for sore throat reduce symptoms at day 3 and the incidence of rheumatic fever and otitis media
}

Del Mar CB, Glasziou PP, Spinks AB. Antibiotics for sore throat. (Cochrane Review, latest version 16 September 1999). In: Cochrane Library. Oxford: Update Software. QUESTION: In primary care settings, are antibiotics effective for adults and children
with acute sore throats?

\section{Data sources}

Studies were identified by searching Medline with the terms pharyngitis, sore throat, and tonsillitis; the Cochrane Library; the Cochrane Acute Respiratory Infections Group trials register; and bibliographies of included studies. Inclusion dates of the studies were 1945-99.

\section{Study selection}

Controlled trials were selected if they compared antibiotics with placebo, if the patients had symptoms of acute sore throat and were in primary care settings, and if outcomes included incidence of acute rheumatic fever within 2 months, acute glomerulonephritis within 1 month, acute otitis media, acute sinusitis, quinsy (peritonsillar abscess), or symptoms of throat soreness, headache, or fever.

\section{Data extraction}

Data were extracted or sought from the authors of trials on study quality, publication date, patient characteristics, antibiotics including duration, outcomes, symptom resolution, and adverse effects.

\section{Main results}

22 studies (10 484 cases of sore throat) met the inclusion criteria. Most were done in the 1950s although 4 were recently published (1996-99). Analyses of all antibiotics together and only trials of penicillin showed reductions in the incidence of acute rheumatic fever; antibiotics also showed modest reductions in the incidence of acute otitis media, quinsy, and symptoms of fever, throat soreness, and headache at day 3 (table). Antibiotics did not prevent acute glomerulonephritis within 2 months or sinusitis within 14 days. Subgroup analyses showed that these results were not changed when blinding, use of antipyretics, confirmed streptococcus infections, or age of the patients were analysed. Data were insufficient to report on adverse effects.

For correspondence: Dr C B Del Mar, Centre for General Practice, University of Queensland Medical School, Brisbane Queensland 4006, Australia.Fax +617 33655442.

\section{Conclusion}

In patients with acute sore throat, antibiotics provide modest benefit for reducing symptoms of throat soreness, fever, and headache at day 3 and the incidence of rheumatic fever, otitis media, and quinsy.

\section{COMMENTARY}

Sore throats are a common complaint in primary health care settings. Antibiotics are frequently used to treat sore throats, especially because of the potential link to rheumatic fever. However, with the decreased incidence of rheumatic fever and increasing concern about antibiotic resistance, the emphasis is on appropriate antibiotic use. For this reason, the systematic review by Del Mar et al will be of particular interest to primary healthcare practitioners.

This review is comprehensive, including studies written in any language dating back to 1945 . However, many of the 22 trials were conducted in the 1950 s, with only 4 published between 1996 and 1999. Many of the studies were of poor quality and their generalisability is questionable. No distinction was made between bacterial and viral sore throats. The most common outcome that the studies examined was rheumatic fever. Only a few trials measured the incidence of otitis media, quinsy (peritonsillar abscess), and sinusitis. Few included a comparison of symptom reduction and the mode of penicillin delivery was not always consistent.

In a recent randomised trial (published after this Cochrane review), Zwart et al compared 7 day with 3 day penicillin treatment for acute sore throat. ${ }^{1}$ They treated patients with at least 3 of the 4 Centor criteria: history of fever, absence of cough, swollen tender anterior cervical lymph nodes, and tonsillar exudate. These are the same criteria used by many practitioners when assessing acute sore throats. In their trial, they concluded that a 7 day course of penicillin was the most effective treatment for adult patients with sore throat caused by Group A streptococci, and possibly those with $3+$ non-group A streptococci. They recommend that reducing duration of treatment should be discouraged. ${ }^{1}$ They also recommend further refinement of current diagnostic tests to ensure prudent use of penicillin.

The review by Del Mar $e t a l$ shows that antibiotics provide a small benefit, shortening duration of symptoms by about 8 hours overall. Factors that practitioners need to keep in mind when deciding whether or not to prescribe antibiotics are incidence of complication rates for the community, the importance of symptom relief and avoidance of complications to the patient and society, costs of antibiotics, adverse effects, and issues related to overuse of antibiotics.

Penny Lee Demarest, RN(EC), BScN Primary Health Care Nurse Practitioner Hunter Street Medical Clinic Woodstock, Ontario, Canada

Zwart S, Sachs AP, Ruijs GJ, et al. BMJ 2000;320:150-4.

Antibiotics $v$ placebo for patients with sore throat in primary care settings (fixed effects model)*

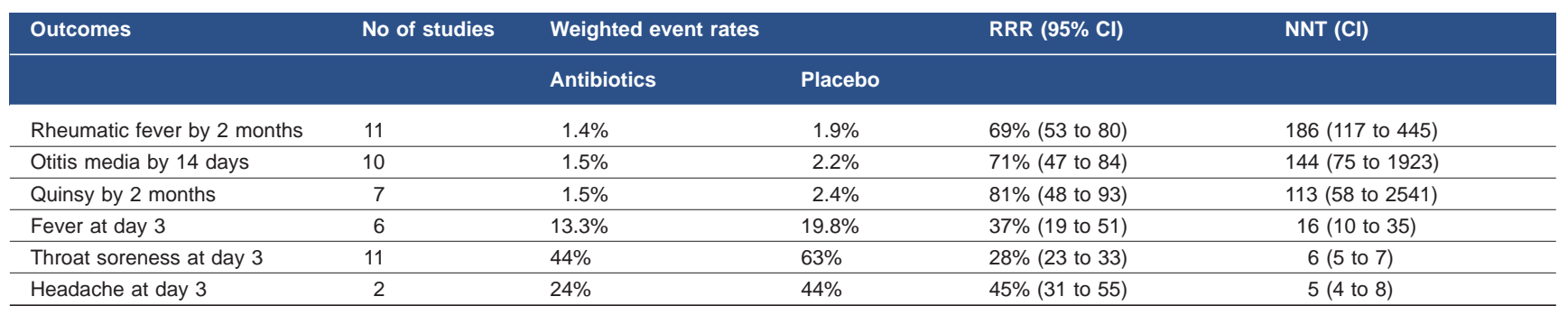

*Abbreviations defined in glossary; RRR, NNT, and $\mathrm{Cl}$ calculated from data in article. 